\title{
Higgs boson mass bounds in the presence of a very heavy fourth quark generation
}

\author{
P. Gerhold, ${ }^{a, b}$ K. Jansen ${ }^{b}$ and J. Kallarackal ${ }^{a, b}$ \\ ${ }^{a}$ Humboldt-Universität zu Berlin, Institut für Physik, \\ Newtonstr. 15, D-12489 Berlin, Germany \\ ${ }^{b} \mathrm{NIC}, \mathrm{DESY}$ \\ Platanenallee 6, D-15738 Zeuthen, Germany \\ E-mail: gerhold@physik.hu-berlin.de, Karl. Jansen@desy.de, \\ Jim.Kallarackal@physik.hu-berlin.de
}

ABSTRACT: We study the effect of a potential fourth quark generation on the upper and lower Higgs boson mass bounds. This investigation is based on the numerical evaluation of a chirally invariant lattice Higgs-Yukawa model emulating the same Higgs-fermion coupling structure as in the Higgs sector of the electroweak Standard Model. In particular, the considered model obeys a Ginsparg-Wilson version of the underlying $\mathrm{SU}(2)_{L} \times \mathrm{U}(1)_{Y}$ symmetry, being a global symmetry here due to the neglection of gauge fields in this model. We present our results on the modification of the upper and lower Higgs boson mass bounds induced by the presence of a hypothetical very heavy fourth quark doublet. Finally, we compare these findings to the standard scenario of three fermion generations.

KeYwords: Higgs Physics, Lattice Quantum Field Theory

ARXIV EPRINT: 1011.1648 


\section{Contents}

$\begin{array}{llr}1 & \text { Introduction } & 1\end{array}$

2 The model 2

$\begin{array}{llr}3 & \text { Simulation strategy } & 4\end{array}$

$\begin{array}{lll}4 & \text { Numerical results } & 6\end{array}$

5 Outlook and conclusions $\quad 8$

\section{Introduction}

The Sakharov explanation for the matter anti-matter asymmetry of the universe suffers from the CP-violating phase of the Standard Model (SM3) falling short by at least 10 orders of magnitude. In addition to this concern the Sakharov picture demands a sufficiently strong first order electroweak phase transition, which is also objected in the framework of the SM3. However, both of the above caveats might be addressable $[1,2]$ by the inclusion of a new fourth fermion generation into an extended version of the Standard Model (SM4). Despite the arguments against the existence of a fourth fermion generation such a scenario nevertheless remains attractive for two reasons. Firstly, there is a strong conceptual interest, since a new fermion generation would need to be very heavy, leading to rather large Yukawa coupling constants and thus to potentially strong interactions with the scalar sector of the theory. Secondly, it has been argued [1,3] (and the references therein) that the fourth fermion generation is actually not excluded by electroweak precision measurements, thus leaving the potential existence of a new fermion generation an open question.

In our contribution, however, we do not present any statement arguing in favour or disfavour of a new fermion generation. Here, we simply assume its existence and focus on the arising consequences on the Higgs boson mass spectrum. With the advent of the LHC this question will become of great phenomenological interest, since the Higgs boson mass bounds, in particular the lower bound, depend significantly on the heaviest fermion mass. Demonstrating this effect will be the main objective of the present work.

Due to the large Yukawa coupling constants of the fourth fermion generation a nonperturbative computation is highly desirable. For this purpose we employ a lattice approach to investigate the strong Higgs-fermion interaction. In fact, we follow here the same lattice strategy that has already been used in ref. [4-6] for the non-perturbative determination of the upper and lower Higgs boson mass bounds in the SM3. This latter approach has the great advantage over the preceding lattice studies of Higgs-Yukawa models (see e.g. refs. [713] and the references therein) that it is the first being based on a consistent formulation 
of an exact lattice chiral symmetry [14], which allows to emulate the chiral character of the Higgs-fermion coupling structure of the Standard Model on the lattice in a conceptually fully controlled manner. The interest in lattice Higgs-Yukawa models has therefore recently been renewed [4-6, 15-20].

The actual details of the considered lattice model will be given in section 2 . In the following section 3 the paper elaborates on the pursued simulation strategy for the determination of the upper and lower Higgs boson mass bounds, i.e. the selection procedure for the bare lattice parameters. The obtained lattice results together with their extrapolations to the infinite volume limit are then presented and discussed in section 4 before the paper ends with some outlook and conclusions in section 5 .

\section{The model}

In order to evaluate the Higgs boson mass bounds we have implemented a lattice model of the pure Higgs-fermion sector of the Standard Model. To be more precise, the Lagrangian of the targeted Euclidean continuum model we have in mind is given as

$$
\begin{aligned}
L_{\mathrm{HY}}= & \bar{t}^{\prime} \not \partial t^{\prime}+\bar{b}^{\prime} \not \partial b^{\prime}+\frac{1}{2} \partial_{\mu} \varphi^{\dagger} \partial_{\mu} \varphi+\frac{1}{2} m_{0}^{2} \varphi^{\dagger} \varphi+\lambda\left(\varphi^{\dagger} \varphi\right)^{2}+y_{b^{\prime}}\left(\bar{t}^{\prime}, \bar{b}^{\prime}\right)_{L} \varphi b_{R}^{\prime}+y_{t^{\prime}}\left(\bar{t}^{\prime}, \bar{b}^{\prime}\right)_{L} \tilde{\varphi} t_{R}^{\prime} \\
& + \text { c.c. of Yukawa interactions, }
\end{aligned}
$$

where we have constrained ourselves to the consideration of the heaviest quark doublet, i.e. the fourth generation doublet, which is labeled here $\left(t^{\prime}, b^{\prime}\right)$. This restriction is reasonable, since the dynamics of the complex scalar doublet $\varphi\left(\tilde{\varphi}=i \tau_{2} \varphi^{*}, \tau_{i}\right.$ : Pauli-matrices $)$ is dominated by the coupling to the heaviest fermions. For the same reason we also neglect any gauge fields in this approach. The quark fields nevertheless have a colour index which actually leads to $N_{c}=3$ identical copies of the fermion doublet appearing in the model. However, for a first exploratory study of the fermionic influence on the Higgs boson mass bounds we have set $N_{c}$ to 1 for simplicity.

Since the Yukawa interaction has a chiral structure, it is important to establish chiral symmetry also in the lattice approach. This has been a long-standing obstacle, which was finally found to be circumventable by constructing the lattice equivalent of $\not \partial$ as well as the left- and right-handed components of the quark fields $t_{L, R}^{\prime}, b_{L, R}^{\prime}$ on the basis of the Neuberger overlap operator $[14,21,22]$. Following the proposition in ref. [14] we have constructed a lattice Higgs-Yukawa model with a global $\mathrm{SU}(2)_{L} \times \mathrm{U}(1)_{Y}$ symmetry.

The fields considered in this model are the aforementioned doublet $\varphi$ as well as $N_{c}$ quark doublets represented by eight-component spinors $\bar{\psi}^{(i)} \equiv\left(\bar{t}^{(i)}, \bar{b}^{\prime(i)}\right), i=1, \ldots, N_{c}$. With $\mathcal{D}^{(o v)}$ denoting the Neuberger overlap operator the fermionic action $S_{F}$ can be written as

$$
S_{F}=\sum_{i=1}^{N_{c}} \bar{\psi}^{(i)} \mathcal{M} \psi^{(i)}, \quad \mathcal{M}=\mathcal{D}^{(o v)}+P_{+} \phi^{\dagger} \operatorname{diag}\left(y_{t^{\prime}}, y_{b^{\prime}}\right) \hat{P}_{+}+P_{-} \operatorname{diag}\left(y_{t^{\prime}}, y_{b^{\prime}}\right) \phi \hat{P}_{-},
$$

where $y_{t^{\prime}, b^{\prime}}$ denote the Yukawa coupling constants and the scalar field $\varphi_{x}$ has been rewritten here as a quaternionic, $2 \times 2$ matrix $\phi_{x}^{\dagger}=\left(\tilde{\varphi}_{x}, \varphi_{x}\right)$, with $x$ denoting the site index of 
the $L_{s}^{3} \times L_{t}$-lattice. The left- and right-handed projection operators $P_{ \pm}$and the modified projectors $\hat{P}_{ \pm}$are given as

$$
P_{ \pm}=\frac{1 \pm \gamma_{5}}{2}, \quad \hat{P}_{ \pm}=\frac{1 \pm \hat{\gamma}_{5}}{2}, \quad \hat{\gamma}_{5}=\gamma_{5}\left(\mathbb{1}-\frac{1}{\rho} \mathcal{D}^{(o v)}\right)
$$

The free Neuberger overlap operator can be written as

$$
\mathcal{D}^{(o v)}=\rho\left\{1+\frac{A}{\sqrt{A^{\dagger} A}}\right\}, \quad A=\mathcal{D}^{(W)}-\rho, \quad 0<\rho<2 r
$$

where $\rho$ is a free, dimensionless parameter within the specified constraints that determines the radius of the circle formed by the eigenvalues of $\mathcal{D}^{(o v)}$ in the complex plane. The operator $\mathcal{D}^{(W)}$ denotes here the Wilson Dirac operator defined as

$$
\mathcal{D}^{(W)}=\sum_{\mu} \gamma_{\mu} \nabla_{\mu}^{s}-\frac{r}{2} \nabla_{\mu}^{b} \nabla_{\mu}^{f}
$$

where $\nabla_{\mu}^{f, b, s}$ are the forward, backward and symmetrized lattice nearest neighbour difference operators in direction $\mu$, while the so-called Wilson parameter $r$ is chosen here to be $r=1$ as usual.

The overlap operator was proven to be local in a field theoretical sense also in the presence of QCD gauge fields at least if the latter fields obey certain smoothness conditions [23, 24]. The locality properties were found to depend on the parameter $\rho$ and the strength of the gauge coupling constant. At vanishing gauge coupling the most local operator was shown to be obtained at $\rho=1$. Here, the notion 'most local' has to be understood in the sense of the most rapid exponential decrease with the distance $|x-y|$ of the coupling strength induced by the matrix elements $\mathcal{D}_{x, y}^{(o v)}$ between the field variables at two remote space-time points $x$ and $y$. For that reason the setting $\rho=1$ will be adopted for the rest of this work.

The introduced action now obeys an exact global $\mathrm{SU}(2)_{L} \times \mathrm{U}(1)_{Y}$ lattice chiral symmetry. For $\Omega_{L} \in \mathrm{SU}(2)$ and $\epsilon \in \mathbb{R}$ the action is invariant under the transformation

$$
\begin{aligned}
\psi & \rightarrow U_{Y} \hat{P}_{+} \psi+U_{Y} \Omega_{L} \hat{P}_{-} \psi, & \bar{\psi} & \rightarrow \bar{\psi} P_{+} \Omega_{L}^{\dagger} U_{Y}^{\dagger}+\bar{\psi} P_{-} U_{Y}^{\dagger}, \\
\phi & \rightarrow U_{Y} \phi \Omega_{L}^{\dagger}, & \phi^{\dagger} & \rightarrow \Omega_{L} \phi^{\dagger} U_{Y}^{\dagger}
\end{aligned}
$$

with the compact notation $U_{Y} \equiv \exp (i \epsilon Y)$ denoting the representations of the global hypercharge symmetry group $\mathrm{U}(1)_{Y}$ for the respective field it is acting on. Particularly, the operator $Y$ yields different hypercharges for the left- and right-handed fields. In the continuum limit eqs. (2.6)-(2.7) eventually recover the (here global) continuum $\mathrm{SU}(2)_{L} \times \mathrm{U}(1)_{Y}$ chiral symmetry.

Finally, the purely bosonic part $S_{\varphi}$ of the total lattice action $S=S_{F}+S_{\varphi}$ is given by the usual lattice $\varphi^{4}$-action

$$
S_{\varphi}=\sum_{x} \frac{1}{2} \nabla_{\mu}^{f} \varphi_{x}^{\dagger} \nabla_{\mu}^{f} \varphi_{x}+\frac{1}{2} m_{0}^{2} \varphi_{x}^{\dagger} \varphi_{x}+\lambda\left(\varphi_{x}^{\dagger} \varphi_{x}\right)^{2}
$$


with the bare mass $m_{0}$, the forward difference operator $\nabla_{\mu}^{f}$ in direction $\mu$, and the bare quartic coupling constant $\lambda$. For the practical lattice implementation, however, a reformulation of eq. (2.8) in terms of the hopping parameter $\kappa$ and the lattice quartic coupling constant $\hat{\lambda}$ proves to be more convenient. It reads

$$
S_{\Phi}=-\kappa \sum_{x, \mu} \Phi_{x}^{\dagger}\left[\Phi_{x+\mu}+\Phi_{x-\mu}\right]+\sum_{x} \Phi_{x}^{\dagger} \Phi_{x}+\hat{\lambda} \sum_{x}\left(\Phi_{x}^{\dagger} \Phi_{x}-N_{c}\right)^{2},
$$

and is fully equivalent to eq. (2.8). This alternative formulation opens the possibility of explicitly studying the limit $\lambda=\infty$ on the lattice. It is further remarked that the appearance of $N_{c}$ in the preceding equation has been adopted here solely for the sake of simplifying the analytical large $N_{c}$ calculation conducted in ref. [18]. The aforementioned connection between eq. (2.8) and eq. (2.9) can be established through a rescaling of the scalar field $\Phi_{x} \in \mathbb{R}^{4}$ and the involved coupling constants according to

$$
\varphi_{x}=\sqrt{2 \kappa}\left(\begin{array}{c}
\Phi_{x}^{2}+i \Phi_{x}^{1} \\
\Phi_{x}^{0}-i \Phi_{x}^{3}
\end{array}\right), \quad \lambda=\frac{\hat{\lambda}}{4 \kappa^{2}}, \quad m_{0}^{2}=\frac{1-2 N_{c} \hat{\lambda}-8 \kappa}{\kappa} .
$$

\section{Simulation strategy}

Due to the triviality of the Higgs sector the targeted Higgs boson mass bounds actually depend on the non-removable, intrinsic cutoff parameter $\Lambda$ of the considered Higgs-Yukawa theory, which can be defined as the inverse lattice spacing, i.e. $\Lambda=1 / a$. To determine these cutoff dependent bounds for a given phenomenological scenario, i.e. for given hypothetical masses of the fourth quark generation, the strategy is to evaluate the maximal interval of Higgs boson masses attainable within the framework of the considered Higgs-Yukawa model being in consistency with this phenomenological setup. The free parameters of the model, being the bare scalar mass $m_{0}$, the bare quartic coupling constant $\lambda$, and the bare Yukawa coupling constants $y_{t^{\prime}, b^{\prime}}$ thus have to be tuned accordingly. The idea for the latter fixation is to employ the assumed fourth generation quark masses $m_{t^{\prime}, b^{\prime}}$ as well as the phenomenological knowledge of the renormalized vacuum expectation value of the scalar field $\varphi$ (vev). The latter is used to determine the physical scale $1 / a$ according to

$$
246 \mathrm{GeV}=\frac{v_{r}}{a} \equiv \frac{v}{\sqrt{Z_{G}} \cdot a},
$$

where $Z_{G}$ denotes the Goldstone renormalization constant and $v$ is the lattice vev.

Concerning the hypothetical masses of the fourth fermion generation quarks, we target here a mass degenerate setup with $m_{t^{\prime}} / a=m_{b^{\prime}} / a=700 \mathrm{GeV}$, which is somewhat above its tree-level unitarity upper bound [25]. The degenerate setting, being unproblematic from a numerical perspective, is owed here to the existence of a fluctuating complex phase in the opposite scenario, i.e. in the non-degenerate case [6]. However, it is remarked that we are currently also investigating a set of other mass settings to study in particular the quark mass dependence of the Higgs boson mass bounds.

In lack of an additional matching condition a one-dimensional freedom is left open here, which can be parametrized in terms of the quartic coupling constant $\lambda$. This freedom finally leads to the emergence of upper and lower bounds on the Higgs boson mass. 
As expected from perturbation theory, one also finds numerically [5] that the lightest and heaviest Higgs boson masses are obtained at vanishing and infinite bare quartic coupling constant, respectively. The lower mass bound will therefore be obtained at $\lambda=0$, while $\lambda=\infty$ will be adjusted to derive the upper bound.

However, in the given lattice model the expectation value $\langle\varphi\rangle$ would always be identical to zero due to the symmetries in eqs. (2.6)-(2.7). The identification $v \equiv\langle\varphi\rangle$ would therefore not yield meaningful results in eq. (3.1). The reason is that the lattice averages over all ground states of the theory, not only over that one which Nature has selected in the broken phase. To study the mechanism of spontaneous symmetry breaking nevertheless, one usually introduces an external current $J$, selecting then only one particular ground state. This current is finally removed after taking the thermodynamic limit, leading then to the existence of symmetric and broken phases with respect to the order parameter $\langle\varphi\rangle$ as desired. An alternative approach, which was shown to be equivalent in the thermodynamic limit [26-28], is to define the vacuum expectation value $v$ as the expectation value of the rotated field $\varphi^{\text {rot }}$ given by a global transformation of the original field $\varphi$ according to

$$
\varphi_{x}^{\mathrm{rot}}=U[\varphi] \varphi_{x}
$$

with the $\mathrm{SU}(2)$ matrix $U[\varphi]$ selected for each configuration of field variables $\left\{\varphi_{x}\right\}$ such that

$$
\sum_{x} \varphi_{x}^{\text {rot }}=\left(\left|\sum_{x}^{0} \varphi_{x}\right|\right) .
$$

Here we use this second approach. According to the notation in eq. (2.8), which already includes a factor $1 / 2$, the relation between the vev $v$ and the expectation value of $\varphi^{\text {rot }}$ is then given as

$$
\left\langle\varphi^{\mathrm{rot}}\right\rangle=\left(\begin{array}{l}
0 \\
v
\end{array}\right) .
$$

In this setup the unrenormalized Higgs mode $h_{x}$ and the Goldstone modes $g_{x}^{1}, g_{x}^{2}, g_{x}^{3}$, can then directly be read out of the rotated field according to

$$
\varphi_{x}^{\mathrm{rot}}=\left(\begin{array}{c}
g_{x}^{2}+i g_{x}^{1} \\
v+h_{x}-i g_{x}^{3}
\end{array}\right) .
$$

The great advantage of this approach is that no limit procedure $J \rightarrow 0$ has to be performed, which simplifies the numerical evaluation of the model tremendously.

The so far lacking prescriptions for calculating the Goldstone renormalization constant $Z_{G}$, the Higgs boson mass $m_{H}$, and the quark masses $m_{t^{\prime}, b^{\prime}}$ have been discussed in detail in ref. [5]. Here it is only stated that $Z_{G}$ is computed from the slope of the inverse Goldstone propagator, the functional form of which has been discussed at one-loop order in ref. [6]. The latter propagator $\tilde{G}_{G}(p)$ is defined as

$$
\begin{aligned}
\tilde{G}_{G}(p) & =\frac{1}{3} \sum_{\alpha=1}^{3}\left\langle\tilde{g}_{p}^{\alpha} \tilde{g}_{-p}^{\alpha}\right\rangle, \\
\tilde{g}_{p}^{\alpha} & =\frac{1}{\sqrt{L_{s}^{3} \cdot L_{t}}} \sum_{x} e^{-i p x} g_{x}^{\alpha}
\end{aligned}
$$


at the discrete lattice momenta $p_{\mu}=2 \pi n_{\mu} / L_{s, t}, n_{\mu}=0, \ldots, L_{s, t}-1$. Correspondingly, the Higgs boson mass $m_{H}$ is derived from the Higgs propagator given as

$$
\begin{aligned}
\tilde{G}_{H}(p) & =\left\langle\tilde{h}_{p} \tilde{h}_{-p}\right\rangle, \\
\tilde{h}_{p} & =\frac{1}{\sqrt{L_{s}^{3} \cdot L_{t}}} \sum_{x} e^{-i p x} h_{x} .
\end{aligned}
$$

Finally, it is remarked that the lattice results on the quark masses presented in this paper have been computed from the decay of the fermionic time correlation functions $C_{f}(\Delta t)$ at large Euclidean time separations $\Delta t$, where $f=t^{\prime}, b^{\prime}$ denotes the quark flavour here. On the lattice the latter time correlation functions can be defined as

$$
C_{f}(\Delta t)=\frac{1}{L_{t} \cdot L_{s}^{6}} \sum_{t=0}^{L_{t}-1} \sum_{\vec{x}, \vec{y}}\left\langle\operatorname{Re} \operatorname{Tr}\left(f_{L, t+\Delta t, \vec{x}} \cdot \bar{f}_{R, t, \vec{y}}\right)\right\rangle,
$$

where the left- and right-handed spinors are given through the projection operators according to

$$
\left(\begin{array}{c}
t^{\prime} \\
b^{\prime}
\end{array}\right)_{L}=\hat{P}_{-}\left(\begin{array}{c}
t^{\prime} \\
b^{\prime}
\end{array}\right) \text { and }\left(\bar{t}^{\prime}, \bar{b}^{\prime}\right)_{R}=\left(\bar{t}^{\prime}, \bar{b}^{\prime}\right) P_{-} .
$$

It is remarked that the given fermionic correlation function would be identical to zero due to the exact lattice chiral symmetry obeyed by the considered Higgs-Yukawa model, if one would not rotate the scalar field $\varphi$ according to eq. (3.2) as discussed above. This rotation is implicitly assumed in the following. Furthermore, it is pointed out that the full all-to-all correlator as defined in eq. (3.10) can be trivially computed, since the sink and source space indices $\vec{x}$ and $\vec{y}$ are summed over independently here. This all-to-all correlator yields very clean signals for the $t^{\prime}, b^{\prime}$ quark mass determination.

\section{Numerical results}

For the eventual determination of the cutoff dependent Higgs boson mass bounds several series of Monte-Carlo calculations have been performed at different values of $\Lambda$ and on different lattice volumes as summarized in table 1 . In order to tame finite volume effects as well as cutoff effects to an acceptable level, we have demanded as a minimal requirement that all particle masses $\hat{m}=m_{H}, m_{t^{\prime}}, m_{b^{\prime}}$ in lattice units fulfill $\hat{m}<0.5$ and $\hat{m} \cdot L_{s, t}>3.5$, at least on the largest investigated lattice volumes. Assuming the Higgs boson mass $m_{H}$ to be around $500-750 \mathrm{GeV}$ this allows to reach cutoff scales between $1500 \mathrm{GeV}$ and $3500 \mathrm{GeV}$ on a $24^{3} \times 32$-lattice. However, despite this setting strong finite volume effects are nevertheless expected induced by the massless Goldstone modes. It is known that these finite size effects are proportional to $1 / L_{s}^{2}$ at leading order [26-28]. An infinite volume extrapolation of the lattice data is therefore mandatory. 


\begin{tabular}{|ccccccc|}
\hline$\kappa$ & $L_{s}$ & $L_{t}$ & $N_{c}$ & $m_{0}^{2}$ & $\lambda$ & $y_{t^{\prime}}=y_{b^{\prime}}$ \\
\hline 0.09442 & $12,16,20,24$ & 32 & 1 & 2.5910 & 0 & 3.2122 \\
0.09485 & $12,16,20,24$ & 32 & 1 & 2.5430 & 0 & 3.2049 \\
0.09545 & $12,16,20,24$ & 32 & 1 & 2.4767 & 0 & 3.1949 \\
0.09560 & $12,16,20,24$ & 32 & 1 & 2.4603 & 0 & 3.1923 \\
0.09605 & $12,16,20,24$ & 32 & 1 & 2.4112 & 0 & 3.1849 \\
\hline 0.21300 & $12,16,20,24$ & 32 & 1 & $\infty$ & $\infty$ & 3.3707 \\
0.21500 & $12,16,20,24$ & 32 & 1 & $\infty$ & $\infty$ & 3.3550 \\
0.22200 & $12,16,20,24$ & 32 & 1 & $\infty$ & $\infty$ & 3.1816 \\
0.22320 & $12,16,20,24$ & 32 & 1 & $\infty$ & $\infty$ & 3.1730 \\
0.22560 & $12,16,20,24$ & 32 & 1 & $\infty$ & $\infty$ & 3.1561 \\
\hline
\end{tabular}

Table 1. The model parameters underlying the lattice calculations performed in this study are presented. The setting $\lambda=0(\lambda=\infty)$ is employed for deriving the lower (upper) Higgs boson mass bound. Depending on the lattice volume the available statistics ranges from $N_{\text {Conf }}=1,000$ to $N_{\text {Conf }}=20,000$.

The finite volume data of the renormalized vacuum expectation value $v_{r}$, the Higgs boson mass $m_{H}$, and the degenerate quark mass $m_{t^{\prime}}=m_{b^{\prime}}$ resulting from the calculations specified in table 1 are presented in figure 1 . These lattice data are plotted versus $1 / L_{s}^{2}$ and extrapolated to the infinite volume limit by means of a linear fit ansatz according to the aforementioned leading order behaviour. Due to the observed curvature arising from the non-leading finite volume corrections only those volumes with $L_{s} \geq 16$ have been respected by the linear fit procedures. One finds that the intended infinite volume extrapolation can indeed reliably be performed thanks to the multitude of investigated lattice volumes reaching from $12^{3} \times 32$ to $24^{3} \times 32$-lattices here.

The quality of the tuning procedure intending to hold the quark masses constant can then be examined in figure $2 \mathrm{~b}$ displaying the results of the infinite volume extrapolation of $m_{t^{\prime}}$. In the considered SM4 scenario the fluctuation of the quark mass has been constrained to roughly $m_{t^{\prime}}=m_{b^{\prime}}=676 \pm 22 \mathrm{GeV}$. For later comparisons with the corresponding SM3 scenario we also present the analogous summary plot of our earlier investigations $[4,5]$ of the latter setup where the degenerate quark masses have been fixed to approximately $m_{t}=m_{b}=173 \pm 3 \mathrm{GeV}$ as demonstrated in figure $2 \mathrm{a}$.

The infinite volume results of the Higgs boson masses are finally presented in figure $3 \mathrm{~b}$. The numerical data for the upper mass bound have moreover been fitted with the analytically expected functional form of the cutoff dependence of the upper Higgs boson mass bound derived in ref. [29]. It is given as

$$
\frac{m_{H}^{\text {up }}}{a}=A_{m} \cdot\left[\log \left(\Lambda^{2} / \mu^{2}\right)+B_{m}\right]^{-1 / 2}
$$

with $A_{m}, B_{m}$ denoting the free fit parameters and $\mu$ being an arbitrary scale, which we have chosen as $\mu=1 \mathrm{TeV}$ here. One learns from this presentation that the obtained results 


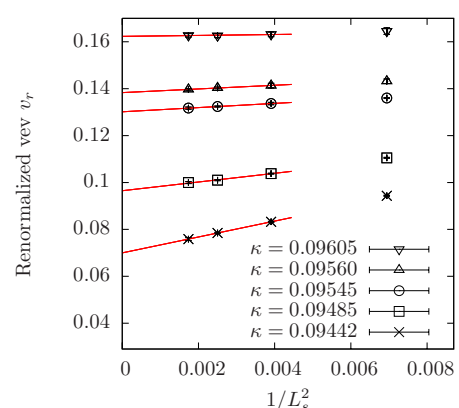

(a)

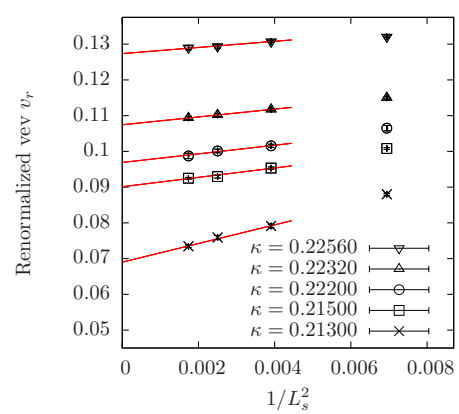

(d)

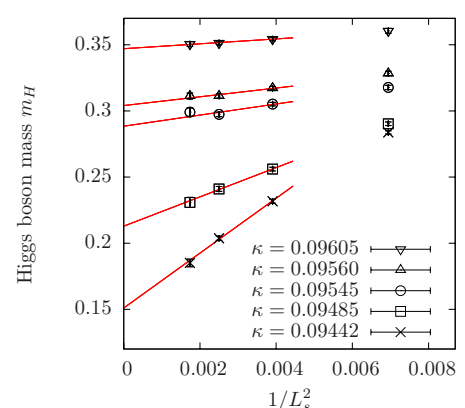

(b)

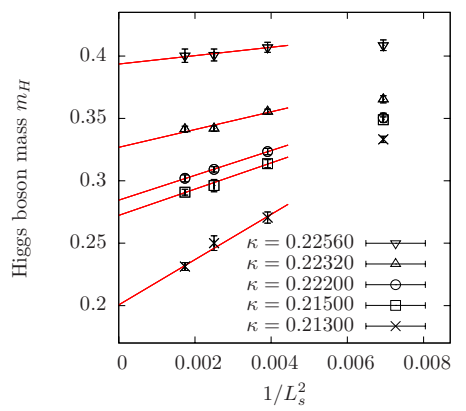

(e)

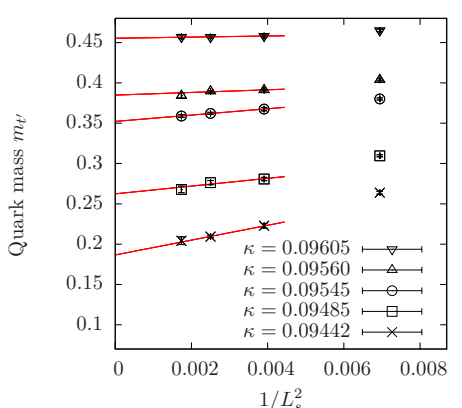

(c)

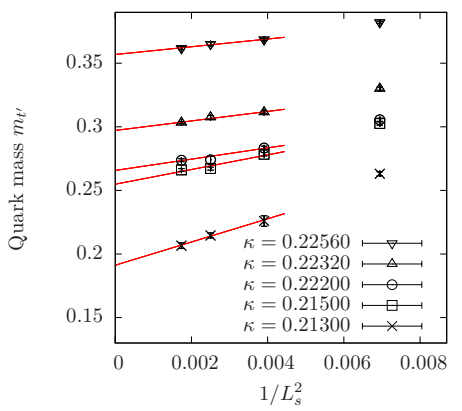

(f)

Figure 1. The finite volume data of the renormalized vacuum expectation value $v_{r}(\mathrm{a}, \mathrm{d})$, the Higgs boson mass $m_{H}(\mathrm{~b}, \mathrm{e})$, and the degenerate quark mass $m_{t^{\prime}}=m_{b^{\prime}}(\mathrm{c}, \mathrm{f})$, as obtained from the lattice calculations specified in table 1 , are plotted versus $1 / L_{s}^{2}$. The upper (lower) row corresponds to the setting $\lambda=0(\lambda=\infty)$. The infinite volume extrapolation is performed by fitting the data to a linear function. Due to the observed curvature arising from the non-leading finite volume corrections only those volumes with $L_{s} \geq 16$ have been respected by the linear fit procedures.

are indeed in good agreement with the expected logarithmic decline of the upper Higgs boson mass bound with increasing cutoff parameter $\Lambda$.

The reader may want to compare these findings to the upper and lower Higgs boson mass bounds previously derived in the SM3. The lattice results corresponding to that setup have been determined in ref. $[4,5]$ and are summarized in figure $3 \mathrm{a}$. The main finding is that especially the lower bound is drastically shifted towards larger values in the presence of the assumed mass-degenerate fourth quark doublet. The upper bound is also significantly increased by the fermionic contributions, however less strongly. From this analysis it seems conclusive that the usually expected light Higgs boson is incompatible with a very heavy fourth fermion generation.

\section{Outlook and conclusions}

The aim of the present work has been the non-perturbative determination of the cutoff dependent upper and lower mass bounds of the Higgs boson assuming the potential existence of a very heavy fourth quark generation. Due to the potentially strong coupling nature of the associated Yukawa coupling constants the lattice approach has been employed here 


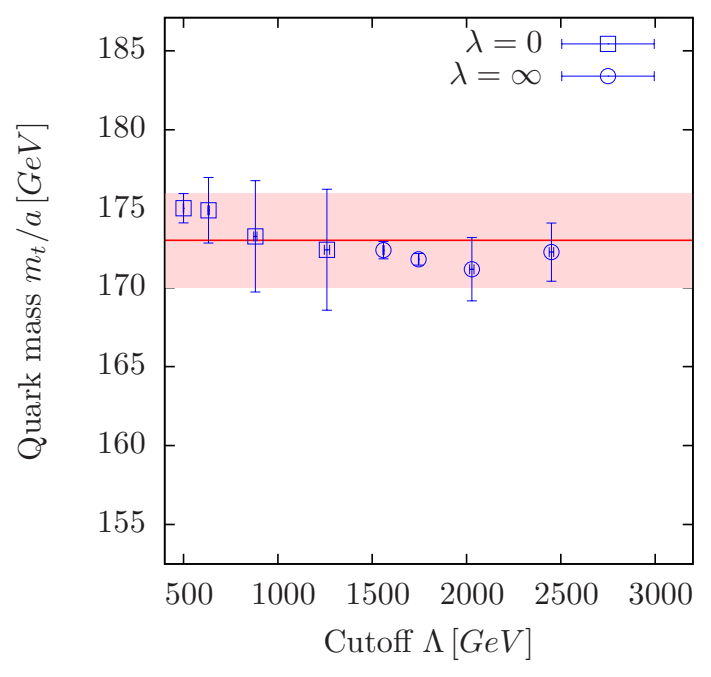

(a)

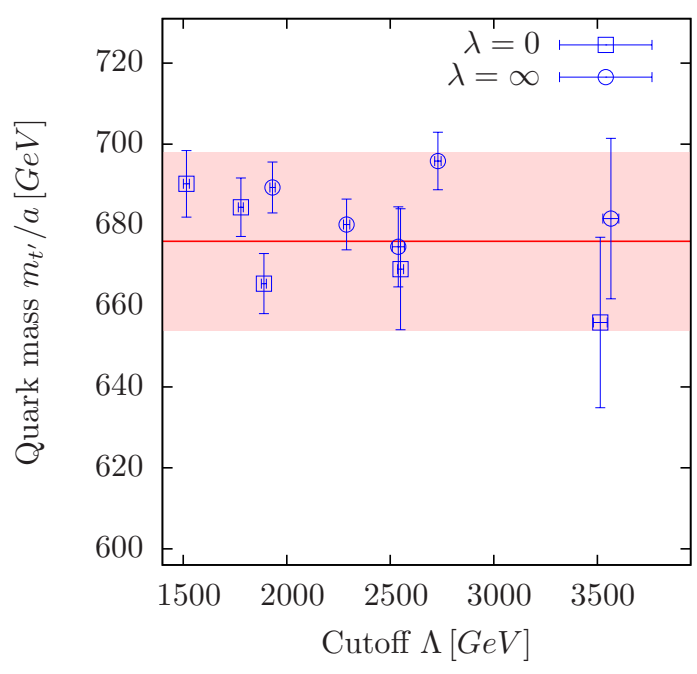

(b)

Figure 2. The infinite volume extrapolations of the degenerate quark masses observed in the lattice calculations specified in table 1 are presented versus the cutoff parameter $\Lambda$. In the SM3 scenario (a) the fluctuation of the quark mass has been constrained to $m_{t}=m_{b}=173 \pm 3 \mathrm{GeV}$, while $m_{t^{\prime}}=m_{b^{\prime}}=676 \pm 22 \mathrm{GeV}$ is adjusted in the SM4 scenario (b).

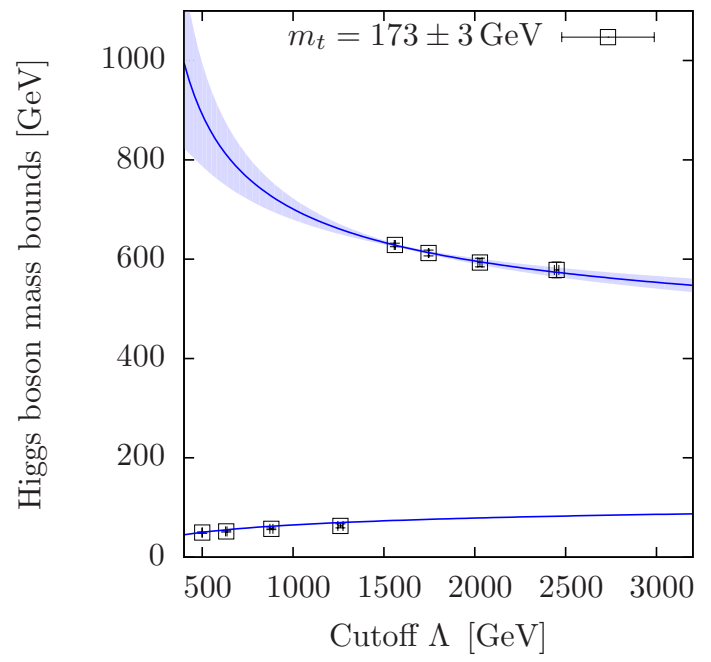

(a)

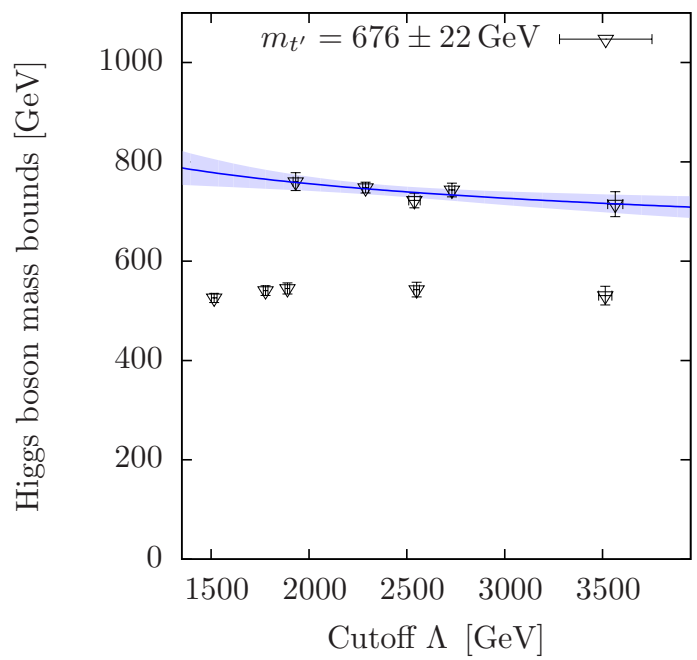

(b)

Figure 3. Upper and lower Higgs boson mass bounds are shown for $N_{c}=1, m_{t}=m_{b}=173 \pm 3 \mathrm{GeV}$ (a) and $N_{c}=1, m_{t^{\prime}}=m_{b^{\prime}}=676 \pm 22 \mathrm{GeV}$ (b). Both upper bounds are each fitted with eq. (4.1). The lower bound in (a) is also compared to a direct analytical computation depicted by the solid line as discussed in ref. [4].

to allow for a non-perturbative investigation of a Higgs-Yukawa model. It serves here as a reasonable simplification of the extended Standard Model SM4, including a fourth fermion generation. The idea is that the considered model contains only those fields and interactions which are most essential for the generation of the Higgs boson mass, i.e. the scalar and quark fields and their mutual interactions. The model has been built on the basis 
of Lüscher's proposal [14] for the construction of chirally invariant lattice Higgs-Yukawa models adapted, however, to the situation of $\varphi$ being a complex doublet equivalent to one Higgs and three Goldstone modes. The resulting chirally invariant lattice Higgs-Yukawa model, based here on the Neuberger overlap operator, then obeys a global $\mathrm{SU}(2)_{\mathrm{L}} \times \mathrm{U}(1)_{\mathrm{Y}}$ symmetry, as desired.

The fundamental strategy underlying the determination of the cutoff dependent Higgs boson mass bounds has then been the numerical evaluation of the maximal interval of Higgs boson masses attainable within the considered Higgs-Yukawa model in consistency with the assumed physical scenario in terms of the masses of the fourth quark generation. Owing to the existence of a fluctuating complex phase in the non-degenerate case [6], the $t^{\prime}, b^{\prime}$ quark masses have been assumed to be degenerate in this work. Here we have considered a hypothetical physical scenario with degenerate fourth generation quark masses at roughly $700 \mathrm{GeV}$. Moreover, we have restricted our focus on $N_{c}=1$ in this work. Since gauge fields are absent in this approach, this simplification does not result in a qualitative modification of the obtained picture. Concerning the quantitative effect of this setting, we expect the Higgs mass to grow further, when the realistic scenario $N_{C}=3$ is adopted, as already observed in some preliminary Monte Carlo runs. The physical scale of the performed lattice calculations has then been fixed by exploiting the phenomenologically known value of the renormalized vacuum expectation value (vev) of the scalar field.

The aforementioned procedure has actually been simplified in this study by exploiting the - perturbatively expected and numerically confirmed [5] - knowledge that the largest attainable Higgs boson masses are indeed observed in the case of an infinite bare quartic coupling constant, while the smallest masses are obtained in the limit of vanishing bare quartic coupling constant. Consequently, the search for the upper and lower Higgs boson mass bounds has been constrained to the bare parameter settings $\lambda=\infty$ and $\lambda=0$, respectively. The resulting finite volume lattice data on the Higgs boson mass, the vacuum expectation value of the scalar field, and the quark masses turned out to be sufficiently precise to allow for their reliable infinite volume extrapolation. The arising extrapolation results are moreover sufficiently precise to resolve their cutoff dependence as demonstrated in figure 3. Concerning the upper bound the obtained cutoff dependence is in good agreement with the analytically expected logarithmic decline and thus with the triviality picture of the Higgs-Yukawa sector. The main finding, however, is that the upper and lower bounds are both shifted towards larger values. While the upper bound is only mildly shifted by roughly $25 \%$, the lower bound is shifted very drastically to roughly $500 \mathrm{GeV}$. From this analysis it seems conclusive that the usually expected light Higgs boson is incompatible with a very heavy fourth fermion generation.

For the future several connected questions should be further investigated. Firstly, it would be interesting to repeat the present study at several other settings of the fourth generation quark masses to compute the quark mass dependence of the Higgs boson mass bounds systematically. This should be done in particular for the lower bound. Moreover, the implemented model allows to tune the bare coupling parameters to arbitrarily large values. It would be of particular interest to determine the largest possible quark mass attainable within the Higgs-Yukawa model beyond a perturbative unitarity consideration. This can be done by evaluating the model in the limit of infinite bare Yukawa coupling constants. 


\section{Acknowledgments}

We thank George Hou and David Lin for ongoing discussions and M. Müller-Preussker for his continuous support. We moreover acknowledge the support of the DFG through the DFG-project Mu932/4-2. The numerical computations have been performed on the HP XC4000 System at the Scientific Supercomputing Center Karlsruhe and on the SGI system $H L R N-I I$ at the HLRN Supercomputing Service Berlin-Hannover.

Open Access. This article is distributed under the terms of the Creative Commons Attribution Noncommercial License which permits any noncommercial use, distribution, and reproduction in any medium, provided the original author(s) and source are credited.

\section{References}

[1] B. Holdom et al., Four statements about the fourth generation, PMC Phys. A 3 (2009) 4 [arXiv:0904.4698] [SPIRES].

[2] M.S. Carena, A. Megevand, M. Quirós and C.E.M. Wagner, Electroweak baryogenesis and new TeV fermions, Nucl. Phys. B 716 (2005) 319 [hep-ph/0410352] [SPIRES].

[3] O. Eberhardt, A. Lenz and J. Rohrwild, Less space for a new family of fermions, Phys. Rev. D 82 (2010) 095006 [arXiv: 1005.3505] [SPIRES].

[4] P. Gerhold and K. Jansen, Lower Higgs boson mass bounds from a chirally invariant lattice Higgs-Yukawa model with overlap fermions, JHEP 07 (2009) 025 [arXiv:0902.4135] [SPIRES].

[5] P. Gerhold and K. Jansen, Upper Higgs boson mass bounds from a chirally invariant lattice Higgs-Yukawa model, JHEP 04 (2010) 094 [arXiv: 1002.4336] [SPIRES].

[6] P. Gerhold, Upper and lower Higgs boson mass bounds from a chirally invariant lattice Higgs-Yukawa model, arXiv:1002.2569 [SPIRES].

[7] J. Smit, Standard model and chiral gauge theories on the lattice, Nucl. Phys. Proc. Suppl. 17 (1990) 3 [SPIRES].

[8] J. Shigemitsu, Higgs-Yukawa chiral models, Nucl. Phys. Proc. Suppl. 20 (1991) 515 [SPIRES].

[9] M.F.L. Golterman, Lattice chiral gauge theories: results and problems, Nucl. Phys. Proc. Suppl. 20 (1991) 528 [SPIRES].

[10] A.K. De and J. Jersák, Yukawa models on the lattice, HLRZ 91-83 preprint edition, HLRZ Jülich, Germany (1991) [Adv. Ser. Direct. High Energy Phys. 10 (1992) 732] [SPIRES].

[11] I. Montvay and G. Münster, Quantum fields on a lattice, Cambridge Monographs on Mathematical Physics, Cambridge University Press, Cambridge U.K. (1997) [SPIRES].

[12] M.F.L. Golterman, D.N. Petcher and E. Rivas, On the Eichten-Preskill proposal for lattice chiral gauge theories, Nucl. Phys. Proc. Suppl. 29BC (1992) 193 [hep-lat/9207005] [SPIRES].

[13] K. Jansen, Domain wall fermions and chiral gauge theories, Phys. Rept. 273 (1996) 1 [hep-lat/9410018] [SPIRES]. 
[14] M. Lüscher, Exact chiral symmetry on the lattice and the Ginsparg-Wilson relation, Phys. Lett. B 428 (1998) 342 [hep-lat/9802011] [SPIRES].

[15] T. Bhattacharya, M.R. Martin and E. Poppitz, Chiral lattice gauge theories from warped domain walls and Ginsparg-Wilson fermions, Phys. Rev. D 74 (2006) 085028 [hep-lat/0605003] [SPIRES].

[16] J. Giedt and E. Poppitz, Chiral lattice gauge theories and the strong coupling dynamics of a Yukawa-Higgs model with Ginsparg-Wilson fermions, JHEP 10 (2007) 076 [hep-lat/0701004] [SPIRES].

[17] E. Poppitz and Y. Shang, Lattice chirality and the decoupling of mirror fermions, JHEP 08 (2007) 081 [arXiv:0706.1043] [SPIRES].

[18] P. Gerhold and K. Jansen, The phase structure of a chirally invariant lattice Higgs-Yukawa model for small and for large values of the Yukawa coupling constant, JHEP 09 (2007) 041 [arXiv:0705.2539] [SPIRES].

[19] P. Gerhold and K. Jansen, The phase structure of a chirally invariant lattice Higgs-Yukawa model - numerical simulations, JHEP 10 (2007) 001 [arXiv:0707.3849] [SPIRES].

[20] Z. Fodor, K. Holland, J. Kuti, D. Nogradi and C. Schroeder, New Higgs physics from the lattice, PoS (LATTICE 2007) 056 [arXiv:0710.3151] [SPIRES].

[21] H. Neuberger, Exactly massless quarks on the lattice, Phys. Lett. B 417 (1998) 141 [hep-lat/9707022] [SPIRES].

[22] H. Neuberger, More about exactly massless quarks on the lattice, Phys. Lett. B 427 (1998) 353 [hep-lat/9801031] [SPIRES].

[23] P. Hernández, K. Jansen and M. Lüscher, Locality properties of Neuberger's lattice Dirac operator, Nucl. Phys. B 552 (1999) 363 [hep-lat/9808010] [SPIRES].

[24] H. Neuberger, Bounds on the Wilson Dirac operator, Phys. Rev. D 61 (2000) 085015 [hep-lat/9911004] [SPIRES].

[25] M.S. Chanowitz, M.A. Furman and I. Hinchliffe, Weak interactions of ultraheavy fermions. 2, Nucl. Phys. B 153 (1979) 402 [SPIRES].

[26] A. Hasenfratz et al., Finite size effects and spontaneously broken symmetries: the case of the O(4) model, Z. Phys. C 46 (1990) 257 [SPIRES].

[27] A. Hasenfratz et al., Goldstone bosons and finite size effects: a numerical study of the $O(4)$ model, Nucl. Phys. B 356 (1991) 332 [SPIRES].

[28] M. Göckeler and H. Leutwyler, Constraint correlation functions in the $O(N)$ model, Nucl. Phys. B 361 (1991) 392 [SPIRES].

[29] M. Lüscher and P. Weisz, Scaling laws and triviality bounds in the lattice $\phi^{4}$ theory. 3. N component model, Nucl. Phys. B 318 (1989) 705 [SPIRES]. 